\title{
Uniformly convex functions II
}

\author{
by Wancang Ma and David Minda (Cincinnati, Ohio)
}

\begin{abstract}
Recently, A. W. Goodman introduced the class UCV of normalized uniformly convex functions. We present some sharp coefficient bounds for functions $f(z)=$ $z+a_{2} z^{2}+a_{3} z^{3}+\ldots \in \mathrm{UCV}$ and their inverses $f^{-1}(w)=w+d_{2} w^{2}+d_{3} w^{3}+\ldots$. The series expansion for $f^{-1}(w)$ converges when $|w|<\varrho_{f}$, where $0<\varrho_{f}$ depends on $f$. The sharp bounds on $\left|a_{n}\right|$ and all extremal functions were known for $n=2$ and 3 ; the extremal functions consist of a certain function $k \in \mathrm{UCV}$ and its rotations. We obtain the sharp bounds on $\left|a_{n}\right|$ and all extremal functions for $n=4,5$, and 6 . The same function $k$ and its rotations remain the only extremals. It is known that $k$ and its rotations cannot provide the sharp bound on $\left|a_{n}\right|$ for $n$ sufficiently large. We also find the sharp estimate on the functional $\left|\mu a_{2}^{2}-a_{3}\right|$ for $-\infty<\mu<\infty$. We give sharp bounds on $\left|d_{n}\right|$ for $n=2,3$ and 4. For $n=2, k^{-1}$ and its rotations are the only extremals. There are different extremal functions for both $n=3$ and $n=4$. Finally, we show that $k$ and its rotations provide the sharp upper bound on $\left|f^{\prime \prime}(z)\right|$ over the class UCV.
\end{abstract}

1. Introduction. This is a continuation of our investigation of uniformly convex functions $[\mathrm{MM}]$. Goodman $[\mathrm{G}]$ introduced the geometrically defined class UCV of uniformly convex functions in the unit disk $\mathbb{D}=\{z:|z|<1\}$. A function $f$ is said to be uniformly convex in $\mathbb{D}$ if $f$ is a normalized $(f(0)=$ $\left.f^{\prime}(0)-1=0\right)$ convex function and has the additional property that for every circular arc $\gamma$ contained in $\mathbb{D}$, with center also in $\mathbb{D}$, the image arc $f(\gamma)$ is convex.

The class CV of normalized $\left(f(0)=f^{\prime}(0)-1=0\right)$ convex univalent functions $f$ is closely related to the class $\mathrm{P}$ of normalized holomorphic functions with positive real part. Recall that a holomorphic function $p$ defined on $\mathbb{D}$ belongs to $\mathrm{P}$ provided that $p(0)=1$ and $\operatorname{Re}\{p(z)\}>0, z \in \mathbb{D}$. Precisely, $f \in \mathrm{CV}$ if and only if $p(z)=1+z f^{\prime \prime}(z) / f^{\prime}(z) \in \mathrm{P}$.

In the earlier paper $[\mathrm{MM}]$, we introduced a subfamily PAR of $\mathrm{P}$ that plays an analogous role for the class UCV. Let $\mathrm{PAR}=\{p(z) \in \mathrm{P}: p(\mathbb{D}) \subseteq \Omega\}$,

1991 Mathematics Subject Classification: Primary 30C45.

Key words and phrases: convex functions, coefficient bounds.

Research partially supported by a National Science Foundation Grant. 
where

$$
\Omega=\left\{w=u+i v: v^{2}<2 u-1\right\}=\{w: \operatorname{Re} w>|w-1|\} .
$$

The characterization that $f \in \mathrm{UCV}$ if and only if $p(z)=1+z f^{\prime \prime}(z) / f^{\prime}(z) \in$ PAR was proved independently by Ma and Minda [MM], and Rønning [R $\left.\varnothing_{1}\right]$. This characterization enabled us to derive some subordination results for the class UCV, from which we derived sharp distortion, growth, rotation and covering theorems, and sharp bounds on the second and third coefficients as well as the sharp order of growth for the coefficients [MM]. In all of these cases, the function $k$ given below is the sole extremal function up to rotations. The sharp bound on the second coefficient and an estimate for all coefficients were also discovered by Rønning $\left[R \emptyset_{1}\right]$. In $\left[R \emptyset_{2}\right]$, Rønning presented a convolution theorem related to UCV.

In $[\mathrm{MM}]$, we also defined holomorphic functions $k_{n}(z)$ in $\mathbb{D}$ by $k_{n}(0)=$ $k_{n}^{\prime}(0)-1=0$ and

$$
1+z k_{n}^{\prime \prime}(z) / k_{n}^{\prime}(z)=q\left(z^{n-1}\right),
$$

where $q(z)$ is a normalized Riemann mapping function from $\mathbb{D}$ to $\Omega$. Explicitly,

$$
\begin{aligned}
q(z) & =1+\frac{2}{\pi^{2}}\left(\log \frac{1+\sqrt{z}}{1-\sqrt{z}}\right)^{2}=1+\sum_{n=1}^{\infty} B_{n} z^{n} \\
& =1+\frac{8}{\pi^{2}} \sum_{n=1}^{\infty}\left(\frac{1}{n} \sum_{k=0}^{n-1} \frac{1}{2 k+1}\right) z^{n} .
\end{aligned}
$$

Clearly $k_{n}(z) \in \mathrm{UCV}$. We write $k_{2}(z)$ as $k(z)$ and set $k(z)=z+A_{2} z^{2}+$ $A_{3} z^{3}+\ldots$ The function $k$ cannot be extremal for the problem of maximizing the modulus of the $n$th coefficient of functions in UCV for $n$ sufficiently large [MM].

In this paper, we first prove that $k$ and its rotations are still extremal for the problem of maximizing the modulus of the $n$th coefficient of functions in UCV when $n=4,5$ and 6 . For the inverse function $f^{-1}(w)=w+d_{2} w^{2}+$ $d_{3} w^{3}+\ldots$, it is interesting to observe that extremal functions of $\left|d_{n}\right|$ are $k_{n}$ and its rotations when $n=2,3$ and 4 . Then we find the sharp estimate of the coefficient functional $\left|\mu a_{2}^{2}-a_{3}\right|,-\infty<\mu<\infty$. Finally, in Section 4, we obtain the sharp upper bound on $\left|f^{\prime \prime}(z)\right|$.

2. Preliminaries. If $f(z)=z+a_{2} z^{2}+a_{3} z^{3}+\ldots \in \mathrm{UCV}$, then there exists a function $p(z)=1+b_{1} z+b_{2} z^{2}+b_{3} z^{3}+\ldots \in$ PAR such that

$$
p(z)=1+z f^{\prime \prime}(z) / f^{\prime}(z) .
$$

Although the bounds $\left|b_{n}\right| \leq B_{1}=8 / \pi^{2}(n=1,2, \ldots)$ [MM] are sharp for $p(z) \in \mathrm{PAR}$, they do not yield sharp bounds for $\left|a_{n}\right|$ when $n \geq 3$. 
Here our idea is to obtain bounds on $\left|a_{n}\right|$ by first expressing $a_{n}$ in terms of coefficients of a function in the class $\mathrm{P}$ and then using coefficient bounds for functions in $\mathrm{P}$. In this section, we derive the expressions needed and prove some coefficient inequalities for functions in $\mathrm{P}$.

The relationship between $f(z) \in \mathrm{UCV}$ and $p(z) \in \mathrm{PAR}$ implies that

$$
n(n-1) a_{n}=\sum_{k=1}^{n-1} k a_{k} b_{n-k}
$$

Furthermore, we have

$$
\begin{gathered}
2 a_{2}=b_{1}, \\
6 a_{3}=b_{2}+b_{1}^{2}, \\
12 a_{4}=b_{3}+\frac{3}{2} b_{2} b_{1}+\frac{1}{2} b_{1}^{3}, \\
20 a_{5}=b_{4}+\frac{4}{3} b_{3} b_{1}+\frac{1}{2} b_{2}^{2}+b_{2} b_{1}^{2}+\frac{1}{6} b_{1}^{4}, \\
30 a_{6}=b_{5}+\frac{5}{4} b_{4} b_{1}+\frac{5}{6} b_{3} b_{2}+\frac{5}{6} b_{3} b_{1}^{2}+\frac{5}{8} b_{2}^{2} b_{1}+\frac{5}{12} b_{2} b_{1}^{3}+\frac{1}{24} b_{1}^{5} .
\end{gathered}
$$

Since $q(z)$ is univalent in $\mathbb{D}$ and $p \prec q$, the function

$$
p_{1}(z)=\frac{1+q^{-1}(p(z))}{1-q^{-1}(p(z))}=1+c_{1} z+c_{2} z^{2}+c_{3} z^{3}+\ldots
$$

is holomorphic and has positive real part in $\mathbb{D}$, that is, $p_{1} \in \mathrm{P}$. Equivalently,

$$
p(z)=q\left(\frac{p_{1}(z)-1}{p_{1}(z)+1}\right) .
$$

From the power series expansion of $q$,

$$
q(z)=1+\frac{8}{\pi^{2}} z+\frac{16}{3 \pi^{2}} z^{2}+\frac{184}{45 \pi^{2}} z^{3}+\frac{352}{105 \pi^{2}} z^{4}+\frac{4504}{1575 \pi^{2}} z^{5}+\ldots
$$

we can express $b_{n}$ in terms of $c_{n}$ by direct calculation. Precisely,

$$
\begin{gathered}
b_{1}=\frac{4}{\pi^{2}} c_{1} \\
b_{2}=\frac{1}{\pi^{2}}\left(4 c_{2}-\frac{2}{3} c_{1}^{2}\right) \\
b_{3}=\frac{1}{\pi^{2}}\left(4 c_{3}-\frac{4}{3} c_{1} c_{2}+\frac{8}{45} c_{1}^{3}\right), \\
b_{4}=\frac{1}{\pi^{2}}\left(4 c_{4}-\frac{4}{3} c_{1} c_{3}-\frac{2}{3} c_{2}^{2}+\frac{8}{15} c_{1}^{2} c_{2}-\frac{2}{35} c_{1}^{4}\right), \\
b_{5}=\frac{1}{\pi^{2}}\left(4 c_{5}-\frac{4}{3} c_{1} c_{4}-\frac{4}{3} c_{2} c_{3}+\frac{8}{15} c_{1}^{2} c_{3}+\frac{8}{15} c_{1} c_{2}^{2}-\frac{8}{35} c_{1}^{3} c_{2}+\frac{32}{1575} c_{1}^{5}\right) .
\end{gathered}
$$

The equalities in (2) and (3) then yield that

$$
2 a_{2}=\frac{4}{\pi^{2}} c_{1}
$$




$$
\begin{gathered}
6 a_{3}=\frac{4}{\pi^{2}} c_{2}+\frac{2}{3 \pi^{2}}\left(\frac{24}{\pi^{2}}-1\right) c_{1}^{2} \\
12 a_{4}=\frac{4}{\pi^{2}} c_{3}+\frac{4}{3 \pi^{2}}\left(\frac{18}{\pi^{2}}-1\right) c_{2} c_{1}+\frac{8}{45 \pi^{2}}\left(1-\frac{45}{2 \pi^{2}}+\frac{180}{\pi^{4}}\right) c_{1}^{3} \\
20 a_{5}=\frac{4}{\pi^{2}} c_{4}+\frac{4}{3 \pi^{2}}\left(\frac{16}{\pi^{2}}-1\right) c_{3} c_{1}+\frac{2}{\pi^{2}}\left(\frac{4}{\pi^{2}}-\frac{1}{3}\right) c_{2}^{2} \\
+\frac{8}{\pi^{2}}\left(\frac{1}{15}-\frac{11}{9 \pi^{2}}+\frac{8}{\pi^{4}}\right) c_{2} c_{1}^{2}+\frac{2}{\pi^{2}}\left(-\frac{1}{35}+\frac{79}{135 \pi^{2}}-\frac{16}{3 \pi^{4}}+\frac{64}{3 \pi^{6}}\right) c_{1}^{4}, \\
30 a_{6}=\frac{4}{\pi^{2}} c_{5}+\frac{4}{\pi^{2}}\left(\frac{5}{\pi^{2}}-\frac{1}{3}\right) c_{4} c_{1}+\frac{4}{3 \pi^{2}}\left(\frac{10}{\pi^{2}}-1\right) c_{3} c_{2} \\
+\frac{4}{3 \pi^{2}}\left(\frac{2}{5}-\frac{20}{3 \pi^{2}}+\frac{40}{\pi^{4}}\right) c_{3} c_{1}^{2}+\frac{2}{\pi^{2}}\left(\frac{4}{15}-\frac{35}{9 \pi^{2}}+\frac{20}{\pi^{4}}\right) c_{2}^{2} c_{1} \\
+\frac{4}{\pi^{2}}\left(-\frac{2}{35}+\frac{1}{\pi^{2}}-\frac{70}{9 \pi^{4}}+\frac{80}{3 \pi^{6}}\right) c_{2} c_{1}^{3} \\
+\frac{2}{3 \pi^{2}}\left(\frac{16}{525}-\frac{109}{189 \pi^{2}}+\frac{47}{9 \pi^{4}}-\frac{80}{3 \pi^{6}}+\frac{64}{\pi^{8}}\right) c_{1}^{5} .
\end{gathered}
$$

Hence coefficient estimates for the class UCV become non-linear coefficient problems for the class P. Note that if $p_{1}(z)=(1+z) /(1-z)$, then $p=q$ and $f=k$. Hence, if $c_{n}=2$ for all $n$, then $b_{n}=B_{n}$ and $a_{n}=A_{n}$. We list the explicit expressions for $A_{n}$ when $n=2,3,4,5$ and 6 :

$$
\begin{gathered}
A_{2}=\frac{4}{\pi^{2}}, \quad A_{3}=\frac{8}{9 \pi^{2}}+\frac{32}{3 \pi^{4}}, \quad A_{4}=\frac{46}{135 \pi^{2}}+\frac{16}{3 \pi^{4}}+\frac{64}{3 \pi^{6}}, \\
A_{5}=\frac{88}{525 \pi^{2}}+\frac{1952}{675 \pi^{4}}+\frac{256}{15 \pi^{6}}+\frac{512}{15 \pi^{8}}, \\
A_{6}=\frac{2252}{23625 \pi^{2}}+\frac{14656}{8505 \pi^{4}}+\frac{4864}{405 \pi^{6}}+\frac{1024}{27 \pi^{8}}+\frac{2048}{45 \pi^{10}} .
\end{gathered}
$$

Now we recall some coefficient bounds for $p_{1}(z)=1+c_{1} z+c_{2} z^{2}+c_{3} z^{3}$ $+\ldots \in \mathrm{P}$. It is well known that $\left|c_{n}\right| \leq 2(n=1,2, \ldots)$. Livingston [L] proved that $\left|c_{n} c_{m}-c_{n+m}\right| \leq 2$ for $n, m=1,2, \ldots$ We can also obtain the following lemma.

LEMMA 1. If $p_{1}(z)=1+c_{1} z+c_{2} z^{2}+c_{3} z^{3}+\ldots \in \mathrm{P}$, then

$$
\begin{gathered}
\left|c_{2 n}-\frac{1}{2} c_{n}^{2}\right| \leq 2-\frac{1}{2}\left|c_{n}\right|^{2}, \\
\left|\mu c_{n}^{2} c_{2 n}-c_{n}^{4}\right| \leq 8(\mu-2) \quad(\mu \geq 4)
\end{gathered}
$$

and

$$
\left|\mu c_{n} c_{2 n}-c_{n}^{3}\right| \leq 4(\mu-2) \quad(\mu \geq 6) .
$$


Proof. For $p_{1}(z)=1+c_{1} z+c_{2} z^{2}+c_{3} z^{3}+\ldots \in P$, we define

$$
h(z)=\frac{1}{n} \sum_{k=1}^{n} p_{1}\left(e^{-i 2 k \pi / n} z\right) \text {. }
$$

Then $h(z)=1+c_{n} z^{n}+c_{2 n} z^{2 n}+c_{3 n} z^{3 n}+\ldots \in P$, so that the function $h_{1}(z)=1+c_{n} z+c_{2 n} z^{2}+c_{3 n} z^{3}+\ldots \in P$. Hence it is enough to show the desired inequalities for $n=1$.

Consider

$$
w(z)=\frac{p_{1}(z)-1}{p_{1}(z)+1}=\frac{1}{2} c_{1} z+\frac{1}{2}\left(c_{2}-\frac{1}{2} c_{1}^{2}\right) z^{2}+\ldots,
$$

which is holomorphic in $\mathbb{D}$ with $w(0)=0$ and $|w(z)|<1$. As $\left|w^{\prime \prime}(0) / 2\right| \leq$ $1-\left|w^{\prime}(0)\right|^{2}[$ A, p. 136], we have

$$
\left|c_{2}-\frac{1}{2} c_{1}^{2}\right| \leq 2-\frac{1}{2}\left|c_{1}\right|^{2} \text {. }
$$

If $\mu \geq 2$, then

$$
\begin{aligned}
\left|\mu c_{1}^{2} c_{2}-c_{1}^{4}\right| & =\left|c_{1}\right|^{2}\left|\mu\left(c_{2}-\frac{1}{2} c_{1}^{2}\right)+\left(\frac{1}{2} \mu-1\right) c_{1}^{2}\right| \\
& \leq\left|c_{1}\right|^{2}\left\{\mu\left(2-\frac{1}{2}\left|c_{1}\right|^{2}\right)+\left(\frac{1}{2} \mu-1\right)\left|c_{1}\right|^{2}\right\}=\left|c_{1}\right|^{2}\left(2 \mu-\left|c_{1}\right|^{2}\right) .
\end{aligned}
$$

As $0 \leq\left|c_{1}\right| \leq 2$ and the right-hand side of the above inequality is an increasing function of $\left|c_{1}\right|$ for $\mu \geq 4$, we have inequality (6) when $n=1$.

If $\mu \geq 2$, then

$$
\begin{aligned}
\left|\mu c_{1} c_{2}-c_{1}^{3}\right| & =\left|c_{1}\right|\left|\mu\left(c_{2}-\frac{1}{2} c_{1}^{2}\right)+\left(\frac{1}{2} \mu-1\right) c_{1}^{2}\right| \\
& \leq\left|c_{1}\right|\left\{\mu\left(2-\frac{1}{2}\left|c_{1}\right|^{2}\right)+\left(\frac{1}{2} \mu-1\right)\left|c_{1}\right|^{2}\right\}=\left|c_{1}\right|\left(2 \mu-\left|c_{1}\right|^{2}\right) .
\end{aligned}
$$

Again the right-hand side of this inequality is an increasing function of $\left|c_{1}\right|$ for $0 \leq\left|c_{1}\right| \leq 2$ and $\mu \geq 6$. This completes the proof of Lemma 1 .

3. Coefficient bounds. Now we are ready to prove coefficient bounds for functions in UCV.

THEOREM 1. Let $f(z)=z+a_{2} z^{2}+a_{3} z^{3}+\ldots \in \mathrm{UCV}$. Then $\left|a_{n}\right| \leq A_{n}$ $(n=4,5,6)$. Equality holds if and only if $f(z)$ is $k(z)$ or one of its rotations.

Proof. When $n=4$, we see that the coefficients in the expression of $a_{4}$ in (4.3) are all positive. So we get $\left|a_{4}\right| \leq A_{4}$ from $\left|c_{k}\right| \leq 2(k=1,2,3)$.

When $n=5$, from (4.4) we have

$$
\begin{aligned}
20 a_{5}= & \frac{4}{\pi^{2}} c_{4}+\frac{4}{3 \pi^{2}}\left(\frac{16}{\pi^{2}}-1\right) c_{3} c_{1}+\frac{2}{\pi^{2}}\left(\frac{4}{\pi^{2}}-\frac{1}{3}\right) c_{2}^{2} \\
& +\frac{16}{3 \pi^{2}}\left(\frac{2}{35}-\frac{43}{45 \pi^{2}}+\frac{4}{\pi^{4}}+\frac{32}{\pi^{6}}\right) c_{2} c_{1}^{2} \\
& +\frac{2}{\pi^{2}}\left(\frac{1}{35}-\frac{79}{135 \pi^{2}}+\frac{16}{3 \pi^{4}}-\frac{64}{3 \pi^{6}}\right)\left(4 c_{1}^{2} c_{2}-c_{1}^{4}\right)
\end{aligned}
$$


In this expression, all coefficients are positive. By using (6) of Lemma 1 for $n=1, \mu=4$ and $\left|c_{k}\right| \leq 2(k=1,2,3,4)$, we see that the upper bound of $\left|a_{5}\right|$ is given if we replace all $c_{k}$ by 2 . That is, we have $\left|a_{5}\right| \leq A_{5}$.

When $n=6$, we rearrange the expression for $a_{6}$ given in (4.5) as follows:

$$
\begin{aligned}
30 a_{6}= & \frac{4}{\pi^{2}} c_{5}+\frac{4}{\pi^{2}}\left(\frac{5}{\pi^{2}}-\frac{1}{3}\right) c_{4} c_{1}+\frac{4}{3 \pi^{2}}\left(\frac{10}{\pi^{2}}-1\right) c_{3} c_{2} \\
& +\frac{2}{3 \pi^{2}}\left(\frac{16}{525}-\frac{109}{189 \pi^{2}}+\frac{47}{9 \pi^{4}}-\frac{80}{3 \pi^{6}}+\frac{64}{\pi^{8}}\right)\left(c_{3}-2 c_{2} c_{1}+c_{1}^{3}\right) c_{1}^{2} \\
& +\frac{2}{3 \pi^{2}}\left(\frac{404}{525}-\frac{2411}{189 \pi^{2}}+\frac{673}{9 \pi^{4}}+\frac{80}{3 \pi^{6}}-\frac{64}{\pi^{8}}\right) c_{3} c_{1}^{2} \\
& +\frac{4}{3 \pi^{2}}\left(\frac{74}{525}-\frac{458}{189 \pi^{2}}+\frac{163}{9 \pi^{4}}-\frac{160}{3 \pi^{6}}-\frac{64}{\pi^{8}}\right)\left(6 c_{2} c_{1}-c_{1}^{3}\right) c_{2} \\
& +\frac{2}{\pi^{2}}\left(-\frac{52}{175}+\frac{1097}{189 \pi^{2}}-\frac{472}{9 \pi^{4}}+\frac{640}{3 \pi^{6}}+\frac{256}{\pi^{8}}\right) c_{2}^{2} c_{1} .
\end{aligned}
$$

Now, all coefficients in this expression are positive. This time we use (7) of Lemma 1 for $n=1$ and $\mu=6,\left|c_{3}-2 c_{2} c_{1}+c_{1}^{3}\right| \leq 2[\mathrm{LZ}]$ and $\left|c_{k}\right| \leq 2$ $(k=1, \ldots, 5)$ to derive that the upper bound of $30\left|a_{6}\right|$ is achieved when we replace all $c_{k}$ by 2 . Thus we have $\left|a_{6}\right| \leq A_{6}$.

In each case, we have used the inequality $\left|c_{1}\right| \leq 2$ in our proof. Hence equality holds only if $p_{1}(z)=(1+z) /(1-z)$ or one of its rotations, which implies that $f=k$ or one of its rotations. On the other hand, it is clear that inequalities become equalities for $k$ and its rotations. This completes the proof of Theorem 1 .

Next we discuss the coefficient functional $\left|\mu a_{2}^{2}-a_{3}\right|$. But first, we introduce the following functions in UCV. For $0 \leq \lambda \leq 1$, define $h_{\lambda}$ and $g_{\lambda}$ by $h_{\lambda}(0)=h_{\lambda}^{\prime}(0)-1=g_{\lambda}(0)=g_{\lambda}^{\prime}(0)-1=0$,

$$
1+\frac{z h_{\lambda}^{\prime \prime}(z)}{h_{\lambda}^{\prime}(z)}=q\left(\frac{z(z+\lambda)}{1+\lambda z}\right), \quad 1+\frac{z g_{\lambda}^{\prime \prime}(z)}{g_{\lambda}^{\prime}(z)}=q\left(-\frac{z(z+\lambda)}{1+\lambda z}\right) .
$$

Then it is clear that both $h_{\lambda}$ and $g_{\lambda}$ belong to UCV. Also notice that $h_{1}=k$, $h_{0}=k_{3}, g_{1}(z)=-k(-z)$ and $g_{0}(z)=-k_{3}(-z)$.

TheOrem 2. Let $f(z)=z+a_{2} z^{2}+a_{3} z^{3}+\ldots \in \mathrm{UCV}$. Then

$$
\left|\mu a_{2}^{2}-a_{3}\right| \leq \begin{cases}\frac{8}{3 \pi^{2}}\left(\frac{6}{\pi^{2}} \mu-\frac{4}{\pi^{2}}-\frac{1}{3}\right) & \text { if } \frac{2}{3}+\frac{5 \pi^{2}}{36} \leq \mu \\ \frac{4}{3 \pi^{2}} & \text { if } \frac{2}{3}-\frac{\pi^{2}}{36} \leq \mu \leq \frac{2}{3}+\frac{5 \pi^{2}}{36} \\ \frac{8}{3 \pi^{2}}\left(-\frac{6}{\pi^{2}} \mu+\frac{4}{\pi^{2}}+\frac{1}{3}\right) & \text { if } \mu \leq \frac{2}{3}-\frac{\pi^{2}}{36} .\end{cases}
$$


Equality holds if and only if $f$ is $k$ or one of its rotations when $\mu<2 / 3-$ $\pi^{2} / 36$ or $2 / 3+5 \pi^{2} / 36<\mu$. For $2 / 3-\pi^{2} / 36<\mu<2 / 3+5 \pi^{2} / 36$, equality holds if and only if $f$ is equal to $k_{3}$ or one of its rotations. If $\mu=2 / 3-$ $\pi^{2} / 36$, then equality holds if and only if $f$ is $h_{\lambda}$ or one of its rotations. Finally, equality holds if and only if $f$ is $g_{\lambda}$ or one of its rotations when $\mu=2 / 3+5 \pi^{2} / 36$.

Re mark. When $2 / 3-\pi^{2} / 36<\mu<2 / 3+5 \pi^{2} / 36$, the above inequality can be improved as follows:

$$
\left|\mu a_{2}^{2}-a_{3}\right|+\frac{1}{12}\left(\frac{\pi^{2}}{3}+12 \mu-8\right)\left|a_{2}\right|^{2} \leq \frac{4}{3 \pi^{2}} \quad \text { if } \frac{2}{3}-\frac{\pi^{2}}{36}<\mu \leq \frac{2}{3}+\frac{\pi^{2}}{18}
$$

and

$$
\left|\mu a_{2}^{2}-a_{3}\right|+\frac{1}{12}\left(\frac{5 \pi^{2}}{3}-12 \mu+8\right)\left|a_{2}\right|^{2} \leq \frac{4}{3 \pi^{2}} \quad \text { if } \frac{2}{3}+\frac{\pi^{2}}{18} \leq \mu<\frac{2}{3}+\frac{5 \pi^{2}}{36} .
$$

In particular, by setting $\mu=1$, we get

$$
\left|a_{2}^{2}-a_{3}\right|+\left(\frac{1}{3}+\frac{\pi^{2}}{36}\right)\left|a_{2}\right|^{2} \leq \frac{4}{3 \pi^{2}} .
$$

This clearly improves $\left|a_{2}^{2}-a_{3}\right|+\frac{1}{3}\left|a_{2}\right|^{2} \leq \frac{1}{3}$, which was established for normalized convex functions (see $[\mathrm{T}]$ ).

Proof of Theorem 2. From (4.1) and (4.2) we obtain

$$
\mu a_{2}^{2}-a_{3}=\frac{1}{3 \pi^{2}}\left(\left(\frac{12}{\pi^{2}} \mu-\frac{8}{\pi^{2}}+\frac{1}{3}\right) c_{1}^{2}-2 c_{2}\right) .
$$

If $\mu \geq 2 / 3+5 \pi^{2} / 36$, then $12 \mu / \pi^{2}-8 / \pi^{2}-5 / 3 \geq 0$ and

$$
\begin{aligned}
\left|\mu a_{2}^{2}-a_{3}\right| & =\frac{1}{3 \pi^{2}}\left|\left(\frac{12}{\pi^{2}} \mu-\frac{8}{\pi^{2}}-\frac{5}{3}\right) c_{1}^{2}+2\left(c_{1}^{2}-c_{2}\right)\right| \\
& \leq \frac{1}{3 \pi^{2}}\left(4\left(\frac{12}{\pi^{2}} \mu-\frac{8}{\pi^{2}}-\frac{5}{3}\right)+4\right)=\frac{8}{3 \pi^{2}}\left(\frac{6}{\pi^{2}} \mu-\frac{4}{\pi^{2}}-\frac{1}{3}\right) .
\end{aligned}
$$

Here we have used $\left|c_{1}^{2}-c_{2}\right| \leq 2$ and $\left|c_{1}\right| \leq 2$.

If $\mu \leq 2 / 3-\pi^{2} / 36$, then $-12 \mu / \pi^{2}+8 / \pi^{2}-1 / 3 \geq 0$ and $\left|c_{1}\right| \leq 2,\left|c_{2}\right| \leq 2$ imply that

$$
\begin{aligned}
\left|\mu a_{2}^{2}-a_{3}\right| & =\frac{1}{3 \pi^{2}}\left|\left(-\frac{12}{\pi^{2}} \mu+\frac{8}{\pi^{2}}-\frac{1}{3}\right) c_{1}^{2}+2 c_{2}\right| \\
& \leq \frac{8}{3 \pi^{2}}\left(-\frac{6}{\pi^{2}} \mu+\frac{4}{\pi^{2}}+\frac{1}{3}\right) .
\end{aligned}
$$


Now we assume $2 / 3-\pi^{2} / 36 \leq \mu \leq 2 / 3+\pi^{2} / 18$, then $-12 \mu / \pi^{2}+8 / \pi^{2}+$ $2 / 3 \geq 0$. By using (5) of Lemma 1 for $n=1$, we have

$$
\begin{aligned}
\mid \mu a_{2}^{2}- & \left.a_{3}\left|+\frac{1}{12}\left(\frac{\pi^{2}}{3}+12 \mu-8\right)\right| a_{2}\right|^{2} \\
= & \frac{1}{3 \pi^{2}}\left\{\left|2\left(c_{2}-\frac{1}{2} c_{1}^{2}\right)+\left(-\frac{12}{\pi^{2}} \mu+\frac{8}{\pi^{2}}+\frac{2}{3}\right) c_{1}^{2}\right|\right. \\
& \left.+\left(\frac{12}{\pi^{2}} \mu-\frac{8}{\pi^{2}}+\frac{1}{3}\right)\left|c_{1}\right|^{2}\right\} \\
\leq & \frac{1}{3 \pi^{2}}\left\{4-\left|c_{1}\right|^{2}+\left(-\frac{12}{\pi^{2}} \mu+\frac{8}{\pi^{2}}+\frac{2}{3}\right)\left|c_{1}\right|^{2}+\left(\frac{12}{\pi^{2}} \mu-\frac{8}{\pi^{2}}+\frac{1}{3}\right)\left|c_{1}\right|^{2}\right\} \\
= & \frac{4}{3 \pi^{2}} .
\end{aligned}
$$

This is the stronger result in the remark above.

Finally, we consider the case when $2 / 3+\pi^{2} / 18 \leq \mu \leq 2 / 3+5 \pi^{2} / 36$. Note that in this case $12 \mu / \pi^{2}-8 / \pi^{2}-2 / 3 \geq 0$. Once again we use (5) for $n=1$ to get

$$
\begin{aligned}
\mid \mu a_{2}^{2}- & \left.a_{3}\left|+\frac{1}{12}\left(\frac{5 \pi^{2}}{3}-12 \mu+8\right)\right| a_{2}\right|^{2} \\
= & \frac{1}{3 \pi^{2}}\left\{\left|2\left(c_{2}-\frac{1}{2} c_{1}^{2}\right)-\left(\frac{12}{\pi^{2}} \mu-\frac{8}{\pi^{2}}-\frac{2}{3}\right) c_{1}^{2}\right|\right. \\
& \left.+\left(-\frac{12}{\pi^{2}} \mu+\frac{8}{\pi^{2}}+\frac{5}{3}\right)\left|c_{1}\right|^{2}\right\} \\
\leq & \frac{1}{3 \pi^{2}}\left\{4-\left|c_{1}\right|^{2}+\left(\frac{12}{\pi^{2}} \mu-\frac{8}{\pi^{2}}-\frac{2}{3}\right)\left|c_{1}\right|^{2}+\left(-\frac{12}{\pi^{2}} \mu+\frac{8}{\pi^{2}}+\frac{5}{3}\right)\left|c_{1}\right|^{2}\right\} \\
= & \frac{4}{3 \pi^{2}} .
\end{aligned}
$$

It is the stronger result stated in the above remark.

From our proof, we see that when $\mu<2 / 3-\pi^{2} / 36$ or $\mu>2 / 3+5 \pi^{2} / 36$, equality holds if and only if $\left|c_{1}\right|=2$, equivalently, $f$ is $k$ or one of its rotations. For $2 / 3-\pi^{2} / 36<\mu<2 / 3+5 \pi^{2} / 36$, equality holds if and only if $\left|c_{2}\right|=2$ and $\left|c_{1}\right|=0$, that is, $f$ is equal to $k_{3}$ or one of its rotations.

If $\mu=2 / 3-\pi^{2} / 36$, then equality holds if and only if $\left|c_{2}\right|=2$, or up to rotation $[\mathrm{P}, \mathrm{p} .41]$,

$$
p_{1}(z)=\frac{1+\lambda}{2} \frac{1+z}{1-z}+\frac{1-\lambda}{2} \frac{1-z}{1+z}, \quad 0 \leq \lambda \leq 1 .
$$

So $f$ is $h_{\lambda}$ or one of its rotations. 
Finally, when $\mu=2 / 3+5 \pi^{2} / 36$, equality holds if and only if $\left|c_{1}^{2}-c_{2}\right|=2$. Thus up to rotation, $p_{1}$ is given by

$$
p_{1}(z)^{-1}=\frac{1+\lambda}{2} \frac{1+z}{1-z}+\frac{1-\lambda}{2} \frac{1-z}{1+z}, \quad 0 \leq \lambda \leq 1 .
$$

This implies that $f$ is $g_{\lambda}$ or one of its rotations. This completes the proof of Theorem 2.

The known sharp bounds on $\left|a_{2}\right|$ and $\left|a_{3}\right|$ can easily be obtained from Theorem 2.

To discuss coefficient bounds for the inverses of functions in UCV, we first observe that for the inverse function $K_{n}(w)$ of $k_{n}(z)$,

$$
K_{n}(w)=w-\frac{8}{(n-1) n \pi^{2}} w^{n}+\ldots .
$$

Hence for $f \in \mathrm{UCV}$ with $F(w)=f^{-1}(w)=w+d_{2} w^{2}+d_{3} w^{3}+\ldots$,

$$
\max \left\{\left|d_{n}\right|: f \in \mathrm{UCV}\right\} \geq \frac{8}{(n-1) n \pi^{2}} .
$$

For $n=2,3,4$, we can prove that equality holds. Note that the series expansion for $f^{-1}(w)$ converges when $|w|<\varrho_{f}$, where $0<-k(-1) \leq \varrho_{f}[\mathrm{MM}]$ depends on $f$.

Theorem 3. Let $f \in \mathrm{UCV}$ and $F(w)=f^{-1}(w)=w+d_{2} w^{2}+d_{3} w^{3}+\ldots$. Then

$$
\left|d_{n}\right| \leq \frac{8}{(n-1) n \pi^{2}} \quad(n=2,3,4) .
$$

Equality holds if and only if $f$ is equal to $k_{n}$ or one of its rotations.

Proof. As $F(f(z))=z$, we have

$$
d_{2}=-a_{2}, \quad d_{3}=2 a_{2}^{2}-a_{3}, \quad d_{4}=-a_{4}+5 a_{3} a_{2}-5 a_{2}^{3} .
$$

By using (4.1)-(4.3), we can express $d_{n}$ in terms of $c_{n}$ as follows:

$$
d_{2}=-\frac{2}{\pi^{2}} c_{1}, \quad d_{3}=\frac{1}{3 \pi^{2}}\left(\left(\frac{16}{\pi^{2}}+\frac{1}{3}\right) c_{1}^{2}-2 c_{2}\right)
$$

and

$$
d_{4}=-\frac{1}{3 \pi^{2}}\left(c_{3}-\left(\frac{1}{3}+\frac{14}{\pi^{2}}\right) c_{2} c_{1}+\left(\frac{2}{45}+\frac{7}{3 \pi^{2}}+\frac{48}{\pi^{4}}\right) c_{1}^{3}\right) .
$$

Now it is clear that $\left|d_{2}\right| \leq 4 / \pi^{2}$. Equality holds if and only if $\left|c_{1}\right|=2$, that is, $f$ is $k$ or one of its rotations.

Also,

$$
\left|d_{3}\right|=\frac{1}{3 \pi^{2}}\left|\left(\frac{16}{\pi^{2}}+\frac{1}{3}\right)\left(c_{2}-c_{1}^{2}\right)+\left(\frac{5}{3}-\frac{16}{\pi^{2}}\right) c_{2}\right| \leq \frac{4}{3 \pi^{2}} .
$$


Here we have used $\left|c_{2}-c_{1}^{2}\right| \leq 2$ and $\left|c_{2}\right| \leq 2$. Equality holds if and only if $\left|c_{2}\right|=2$ and $\left|c_{1}\right|=0$, or equivalently, $f$ is $k_{3}$ or one of its rotations.

By using $\left|c_{3}-2 c_{2} c_{1}+c_{1}^{3}\right| \leq 2[\mathrm{LZ}],\left|c_{3}-c_{2} c_{1}\right| \leq 2$ and $\left|c_{3}\right| \leq 2$, we get

$$
\begin{aligned}
\left|d_{4}\right|= & \frac{1}{3 \pi^{2}} \mid\left(\frac{2}{45}+\frac{7}{3 \pi^{2}}+\frac{48}{\pi^{4}}\right)\left(c_{3}-2 c_{2} c_{1}+c_{1}^{3}\right) \\
& +\left(\frac{11}{45}+\frac{28}{3 \pi^{2}}-\frac{96}{\pi^{4}}\right)\left(c_{3}-c_{2} c_{1}\right)+\left(\frac{32}{45}-\frac{35}{3 \pi^{2}}+\frac{48}{\pi^{4}}\right) c_{3} \mid \leq \frac{2}{3 \pi^{2}} .
\end{aligned}
$$

In this case, the inequality becomes equality if and only if $\left|c_{3}\right|=2$ and $\left|c_{2}\right|=\left|c_{1}\right|=0$, which is the same as saying $f$ is $k_{4}$ or one of its rotations. The proof of Theorem 3 is now complete.

4. Upper bound on $\left|f^{\prime \prime}(z)\right|$. Finally in this section, we derive the sharp upper bound on $\left|f^{\prime \prime}(z)\right|$ for functions in UCV.

THEOREM 4. Let $f \in \mathrm{UCV}$ and $|z|=r<1$. Then

$$
\left|f^{\prime \prime}(z)\right| \leq k^{\prime \prime}(r) \text {. }
$$

Equality holds for any $z \in \mathbb{D}$ if and only if $f$ is $k$ or one of its rotations.

P r o of. Let $p(z)=1+z f^{\prime \prime}(z) / f^{\prime}(z)$. Then $p \prec q$ implies that $p-1 \prec q-1$. As all coefficients of $q-1$ are positive, the subordination principle yields that for $|z|=r$,

$$
|p(z)-1| \leq q(r)-1
$$

This is the same as

$$
\left|f^{\prime \prime}(z) / f^{\prime}(z)\right| \leq k^{\prime \prime}(r) / k^{\prime}(r) .
$$

From $\left|f^{\prime}(z)\right| \leq k^{\prime}(r)[\mathrm{MM}]$, we see that

$$
\left|f^{\prime \prime}(z)\right| \leq k^{\prime}(r)\left|f^{\prime \prime}(z) / f^{\prime}(z)\right| \leq k^{\prime \prime}(r) .
$$

We also know that equality holds in $\left|f^{\prime}(z)\right| \leq k^{\prime}(r)$ for some $z \neq 0$ if and only if $f$ is a rotation of $k$ [MM]. Moreover, equality in Theorem 4 at $z=0$ is equivalent to $\left|a_{2}\right|=A_{2}$. Hence equality holds if and only if $f$ is $k$ or one of its rotations. This completes our proof.

\section{References}

[A] L. V. Ahlfors, Complex Analysis, 3rd ed., McGraw-Hill, New York, 1979.

[G] A. W. Goodman, On uniformly convex functions, Ann. Polon. Math. 56 (1991), 87-92.

[L] A. E. Livingston, The coefficients of multivalent close-to-convex functions, Proc. Amer. Math. Soc. 21 (1969), 545-552.

[LZ] R. J. Libera and E. J. Złotkiewicz, Early coefficients of the inverse of a regular convex function, Proc. Amer. Math. Soc. 85 (1982), 225-230. 
[MM] W. Ma and D. Minda, Uniformly convex functions, Ann. Polon. Math. 57 (1992), 165-175.

[P] Ch. Pommerenke, Univalent Functions, Vandenhoeck \& Ruprecht, Göttingen, 1975.

$\left[\mathrm{R} \varnothing_{1}\right] \quad \mathrm{F} . \mathrm{R} \varnothing \mathrm{nning}$, Uniformly convex functions and a corresponding class of starlike functions, Proc. Amer. Math. Soc. 118 (1993), 189-196.

$\left[\mathrm{R} \varnothing_{2}\right]-$, On starlike functions associated with parabolic regions, Ann. Univ. Mariae Curie-Skłodowska Sect. A 45 (1991), 117-122.

[T] S. Y. Trimble, A coefficient inequality for convex univalent functions, Proc. Amer. Math. Soc. 48 (1975), 266-267.

DEPARTMENT OF MATHEMATICAL SCIENCES

UNIVERSITY OF CINCINNATI

CINCINNATI, OHIO 45221-0025

U.S.A.

Reçu par la Rédaction le 28.8.1992

Révisé le 1.2.1993 